\title{
EXISTENCE OF MULTIPLE POSITIVE SOLUTIONS FOR A VARIATIONAL INEQUALITY OF KIRCHHOFF TYPE
}

\section{BELAL ALMUAALEMI, HAIBO CHEN and SOFIANE KHOUTIR}

School of Mathematics and Statistics

Central South University

Changsha, Hunan 410083

P. R. China

e-mail: belal_math@csu.edu.cn

math_chb@csu.edu.cn

sofiane_math@csu.edu.cn

\begin{abstract}
This paper is devoted to prove the existence and the multiplicity of positive solutions for a class of a nonlinear variational inequality of Kirchhoff type. Under more general superlinear assumptions on the nonlinear term, we prove the existence of multiple positive solutions via non-smooth critical point theory for Szulkin-type functional.
\end{abstract}

2010 Mathematics Subject Classification: 35J87, 49J40.

Keywords and phrases: variational inequality, multiple positive solutions, Szulkin-type functional.

This work was supported by Natural Science Foundation of China (11271372) and the Mathematics and Interdisciplinary Science Project of CSU.

Received November 2, 2017

(ㄷ) 2018 Scientific Advances Publishers 


\section{Introduction and Main Results}

Consider the following variational inequality of Kirchhoff type:

$$
\begin{cases}-\left(a+b \int_{\Omega}|\nabla u|^{2} d x\right) \Delta u \geq f(x, u), & \text { in } \Omega, \\ u=0, & \text { on } \partial \Omega,\end{cases}
$$

where $\Omega$ is smooth bounded domain in $\mathbb{R}^{N}(N=1,2$, or 3$), a, b>0$ are constants and $f: \Omega \times \mathbb{R} \rightarrow \mathbb{R}$ is continuos and satisfies:

$\left(\mathrm{f}_{1}\right) f \in C(\Omega \times \mathbb{R}, \mathbb{R})$ and there exist $c>0$ and $p \in\left(1,2^{*}\right)\left(2^{*}=\frac{2 N}{N-2}\right.$ if $N \geq 3,2^{*}=+\infty$ if $N<3$, is the Sobolev critical exponent) such that

$$
|f(x, u)| \leq c\left(1+|u|^{p-1}\right), \quad \forall(x, u) \in \Omega \times \mathbb{R} .
$$

$\left(\mathrm{f}_{2}\right) \frac{F(x, u)}{|u|^{4}} \rightarrow+\infty$ as $|u| \rightarrow+\infty$ uniformly in $x \in \Omega$ and there exists $r_{0}>0$ such that

$$
F(x, u) \geq 0, \quad \forall(x, u) \in \Omega \times \mathbb{R},|u| \geq r_{0},
$$

where $F(x, u)=\int_{0}^{u} f(x, s) d s$.

$\left(f_{3}\right)$ there exists a constant $\beta<a \lambda_{1}$ such that

$$
4 F(x, u) \leq f(x, u) u+\beta|u|^{2}, \quad \forall(x, u) \in \Omega \times \mathbb{R}
$$

where $\lambda_{1}>0$ is the first eigenvalue of $\left(-\Delta, H_{0}^{1}(\Omega)\right)$.

(f $\left.f_{4}\right) \widetilde{F}(x, u) \geq 0$ for all $(x, u) \in \Omega \times \mathbb{R}$, and $\widetilde{F}(x, s) \leq \widetilde{F}(x, t)$ whenever $(s, t) \in \mathbb{R}^{+} \times \mathbb{R}^{+}$and $s \leq t$, where $\tilde{F}(x, u)=\frac{1}{4} f(x, u) u-F(x, u)$.

$$
\left(\mathrm{f}_{5}\right) f(x, u)=-f(x, u) \text { for all }(x, u) \in \Omega \times \mathbb{R} .
$$

Variational inequalities describe phenomena from mathematical physics. They have applications in physics, mechanics, engineering, optimization, and elliptic inequalities, see, for example, [1-5]. 
The aim of this work is to study a Kirchhoff type variational inequality which is defined on $\Omega$ by using a non-smooth critical point theory due to Szulkin. In [7], the author has proved a number of existence theorem for critical point of functionals which are not smooth. He has generalized some minimization and minimax methods in critical point theory to a class of functionals which are not necessarily continuous and has introduced a new concept of compactness which is suitable to study these kinds of problems.

In the present paper, by using a minimization principle and the mountain pass theorem of Szulkin-type, we prove existence of positive solutions to a variational inequality of Kirchhoff-type in a closed convex set.

Let $K=\left\{u \in H_{0}^{1}(\Omega): u \geq 0\right\}$ be the closed convex set in Sobolev space $H_{0}^{1}(\Omega)$ and we consider the problem, denoted by $(P)$ :

Given $f: \Omega \times \mathbb{R} \rightarrow \mathbb{R}$ a continuous function and $a, b>0$, find $u \in K$ such that

$$
\left(a+b \int_{\Omega}|\nabla u|^{2} d x\right) \int_{\Omega} \nabla u \cdot(\nabla v-\nabla u) d x-\int_{\Omega} f(x, u)(v-u) d x \geq 0, \quad \forall v \in K .
$$

Such kind of problems are called obstacle problems and they have been largely studied due to its physical application. See, for example, the classical books Kinderlehrer and Stampacchia [4], Rodrigues [6], and Troianiello [8] and the references therein.

The main results of this paper are the following:

Theorem 1.1. Assume that $\left(\mathrm{f}_{1}\right),\left(\mathrm{f}_{2}\right),\left(\mathrm{f}_{3}\right)$, and $\left(\mathrm{f}_{5}\right)$ hold. Then problem (1.1) possesses at least one distinct pair of positive solution.

Theorem 1.2. Assume that $\left(\mathrm{f}_{1}\right),\left(\mathrm{f}_{2}\right),\left(\mathrm{f}_{4}\right)$, and $\left(\mathrm{f}_{5}\right)$ hold. Then problem (1.1) possesses at least one distinct pair of positive solution. 
Remark 1.3. Note that conditions $\left(f_{3}\right)$ and $\left(f_{4}\right)$ are weaker than the well-known Ambrosetti-Rabinowitz condition (AR for short), which was first introduced in [10], that is,

$$
\exists \nu>4: \nu F(x, t) \leq t f(x, t), t \neq 0 .
$$

Indeed, there are functionals $f(x, u)$ satisfying conditions $\left(\mathrm{f}_{1}\right)-\left(\mathrm{f}_{5}\right)$, and not satisfying the AR condition. For example, let

$$
F(x, u)=u^{4} \ln \left(1+u^{4}\right)
$$

Then

$$
f(x, u)=4 u^{3} \ln \left(1+u^{4}\right)+4 u^{4} \frac{u^{3}}{1+u^{4}} .
$$

By a simple computation, one can deduces that

$$
\nu F(x, u)-f(x, u) u=(\nu-4) u^{4} \ln \left(1+u^{4}\right)-4 u^{4} \frac{u^{4}}{1+u^{4}}>0
$$

for $|u|$ large enough. Thus, $f$ does not satisfy the AR-condition. Moreover, it is easy to check that $f$ satisfies all the assumptions of Theorems 1.1 and 1.2 .

\section{Variational Framework and Technical Lemmas}

Let $H:=H_{0}^{1}(\Omega)$ be the Sobolev space equipped with the inner product and the norm

$$
\langle u, v\rangle=\int_{\Omega} \nabla u \cdot \nabla v d x, \quad\|u\|=\langle u, u\rangle^{\frac{1}{2}}
$$

We denote by $|\cdot|_{p}$ the usual $L^{p}$-norm. Since $\Omega$ is a bounded domain, then $H \hookrightarrow L^{p}(\Omega)$ continuously for $p \in\left[1,2^{*}\right]$, and compactly for $p \in\left[1,2^{*}\right]$, and there exists $\gamma_{p}>0$ such that

$$
|u|_{p} \leq \gamma_{p}\|u\|, \quad \forall u \in H
$$


Let $K=\{u \in H, u \geq 0\}$ be the closed convex set in the space $H$. Recall that a function $u \in H$ is called weak solution of (1.1) if

$$
\left(a+b \int_{\Omega}|\nabla u|^{2} d x\right) \int_{\Omega} \nabla u \cdot(\nabla v-\nabla u) d x-\int_{\Omega} f(x, u)(v-u) d x \geq 0, \forall v \in H .
$$

Now we give some preliminaries about Szulkin-type function (see [7]). Let $X$ be a real Banach space and $X^{*}$ its dual. Let $\phi$ be a functional which is of class $C^{1}$ and let $\psi: X \rightarrow \mathbb{R} \cup\{+\infty\}$ be a proper (i.e., $\psi \neq+\infty$ ), convex and lower semicontinuous functional. We say that $I=\phi+\psi$ is a Szulkin-type functional. An element $u \in X$ is called a critical point of $I=\phi+\psi$ if

$$
\phi^{\prime}(u)(v-u)+\psi(v)-\psi(u) \geq 0, \quad \text { for all } \quad v \in X,
$$

which is equivalent to

$$
0 \in \phi^{\prime}(u)+\partial \psi(u) \quad \text { in } \quad X^{*}
$$

where $\partial \psi(u)$ is the subdifferential of the convex functional $\psi$ at $u \in X$ defined by

$$
\partial \psi(u)=\left\{\phi \in X^{*}: \psi(v)-\psi(u) \geq\langle\phi, v-u\rangle, \quad \forall v \in X\right\}
$$

Definition 2.1. The functional $I=\phi+\psi$ satisfies the Palais-Smale condition at level $c \in \mathbb{R}$, denoted by $(P S Z)_{c}$ if every sequence $\left\{u_{n}\right\} \subset X$ such that $\lim _{n \rightarrow \infty} I\left(u_{n}\right)=c$ and

$$
\left\langle\phi^{\prime}\left(u_{n}\right), v-u_{n}\right\rangle+\psi(v)-\psi\left(u_{n}\right) \geq-\varepsilon_{n}\left\|v-u_{n}\right\| \quad \text { for all } v \in X,
$$

where $\varepsilon_{n} \rightarrow 0$, possesses a convergent subsequence. 
The following theorem which was proved by Szulkin, is the main tool to prove the main results of this paper.

Theorem 2.2. Let $X$ be a Banach space, $I: X \rightarrow(-\infty,+\infty] a$ Szulkin-type functional satisfies $(P S Z)_{c}, I(0)=0$ and $\phi, \psi$ are even. Assume also that

(i) there exists a subspace $X_{1}$ of $X$ of finite codimension and numbers $\alpha, \rho>0$ such that $\left.I\right|_{\partial B \rho \cap X_{1}} \geq \alpha$

(ii) there is a finite dimensional subspace $X_{2}$ of $X, \operatorname{dim} X_{2}>\operatorname{codim} X_{1}$, such that $I(u) \rightarrow-\infty$ as $\|u\| \rightarrow \infty, u \in X_{2}$.

Then I has at least $\operatorname{dim} X_{2}-\operatorname{codim} X_{1}$ distinct pairs of nontrivial critical points.

We define the functional $\phi: H \rightarrow \mathbb{R}$ by

$$
\phi(u)=\frac{a}{2}\|u\|^{2}+\frac{b}{4}\|u\|^{4}-\int_{\Omega} F(x, u(x)) d x
$$

Using $\left(f_{1}\right)$ and the Sobolev embedding theorem, we can prove easily that $\phi \in C^{1}(H, \mathbb{R})$. We define the indicator functional of the set $K$ by

$$
\psi_{K}(u)= \begin{cases}0, & \text { if } u \in K, \\ +\infty, & \text { if } u \notin K .\end{cases}
$$

We remark that the functional $\psi_{K}$ is convex, proper and lower semicontinuous. So, $I=\phi+\psi_{K}$ is a Szulkin-type functional.

Lemma 2.3. If $u \in H$ is a critical point of $I=\phi+\psi_{K}$, then $u$ is a solution of problem (1.1). 
Proof. Let $u \in H$ be a critical point of $I=\phi+\psi_{K}$. Then, we have

$$
\phi^{\prime}(u)(v-u)+\psi_{K}(v)-\psi_{K}(u) \geq 0, \quad \forall v \in H .
$$

We first prove that $u \in K$. If this were not true, we have $\psi_{K}(u)=+\infty$, and taking $v=0 \in K$ in the above inequality, we obtain a contradiction. Next, for a fixed $v \in K$, since

$$
\begin{aligned}
0 \leq \phi^{\prime}(u)(v-u)= & \left(a+b\|u\|^{2}\right) \int_{\Omega} \nabla u(\nabla v-\nabla u) d x \\
& -\int_{\Omega} f(x, u)(v-u) d x
\end{aligned}
$$

the inequality is proved.

Lemma 2.4. Assume that $f$ satisfies $\left(\mathrm{f}_{1}\right)$ and $\left(\mathrm{f}_{3}\right)$, then $I=\phi+\psi_{K}$ satisfies the $(P S Z)_{c}$ for every $c \in \mathbb{R}$.

Proof. Let $\left\{u_{n}\right\} \subset H$ be such that

$$
I\left(u_{n}\right)=\phi\left(u_{n}\right)+\psi_{K}\left(u_{n}\right) \rightarrow c, \quad(c \in \mathbb{R}),
$$

and

$$
\phi^{\prime}\left(u_{n}\right)\left(v-u_{n}\right)+\psi_{K}(v)-\psi_{K}\left(u_{n}\right) \geq-\varepsilon_{n}\left\|v-u_{n}\right\|, \quad \forall v \in H,
$$

where $\left\{\varepsilon_{n}\right\} \subset[0, \infty\}$ is a sequence with $\varepsilon_{n} \rightarrow 0$. By (2.5), we have $\left\{u_{n}\right\}$ is in $K$. Next, we prove that $\left\{u_{n}\right\}$ is bounded in $H$. It follows from $\left(\mathrm{f}_{3}\right)$ and (2.5) that

$$
\begin{aligned}
c+1+\left\|u_{n}\right\| & \geq \phi\left(u_{n}\right)-\frac{1}{4}\left\langle\phi^{\prime}\left(u_{n}\right), u_{n}\right\rangle \\
& =\frac{a}{4}\left\|u_{n}\right\|^{2}+\int_{\Omega}\left(\frac{1}{4} f\left(x, u_{n}\right) u_{n}-F\left(x, u_{n}\right)\right) d x \\
& \geq \frac{a}{4}\|u\|^{2}-\frac{\beta}{4} \int_{\Omega}|u|^{2} d x \\
& \geq \frac{1}{4}\left(a-\frac{\beta}{\lambda_{1}}\right)\left\|u_{n}\right\|^{2} .
\end{aligned}
$$


Since $\beta<a \lambda_{1}$, then $\left(a-\frac{\beta}{\lambda_{1}}\right)>0$. Thus $\left\{u_{n}\right\}$ is bounded in $H$. Because the sequence $\left\{u_{n}\right\}$ is bounded in $H$, going if necessary to a subsequence, we may assume that

$$
\begin{aligned}
& u_{n} \rightarrow u \quad \text { in } H ; \\
& u_{n} \rightarrow u \quad \text { in } L^{p}(\Omega), \quad \text { for } 1 \leq p<2^{*} ; \\
& u_{n}(x) \rightarrow u(x) \quad \text { a.e. } x \in \Omega .
\end{aligned}
$$

As $K$ is weakly closed, then $u \in K$. Setting $v=u$ in (2.6), we obtain that

$$
\begin{aligned}
(a & \left.+b\left\|u_{n}\right\|^{2}\right) \int_{\Omega} \nabla u_{n}\left(\nabla u-\nabla u_{n}\right) d x \\
& +\int_{\Omega} f\left(x, u_{n}\right)\left(u-u_{n}\right) d x \geq-\varepsilon_{n}\left\|u-u_{n}\right\| .
\end{aligned}
$$

Therefore, for large $n \in \mathbb{N}$, we have

$$
\begin{aligned}
\left(a+b\left\|u_{n}\right\|^{2}\right)\left\|u-u_{n}\right\|^{2} \leq & \left(a+b\left\|u_{n}\right\|^{2}\right) \int_{\Omega} \nabla u\left(\nabla u-\nabla u_{n}\right) d x \\
& +\int_{\Omega} f\left(x, u_{n}\right)\left(u-u_{n}\right) d x+\varepsilon_{n}\left\|u-u_{n}\right\| .
\end{aligned}
$$

In one hand, by $\left(f_{1}\right),(2.7)$ and the Hölder inequality, one has

$$
\begin{aligned}
\int_{\Omega} f\left(x, u_{n}\right)\left(u-u_{n}\right) d x & \leq \int_{\Omega} c\left(1+\left|u_{n}\right|^{p-1}\right)\left(u-u_{n}\right) d x \\
& \leq c \int_{\Omega}\left|u-u_{n}\right| d x+c \int_{\Omega}\left|u_{n}\right|^{p-1}\left|u-u_{n}\right| d x \\
& \leq c\left\|u-u_{n}\right\|_{1}+c\left\|u-u_{n}\right\|_{p}\left\|u_{n}\right\|_{p}^{p-1} \\
& \rightarrow 0 \quad \text { as } \quad n \rightarrow \infty .
\end{aligned}
$$


In the other hand, by (2.7) and the fact that $\left\{u_{n}\right\}$ is bounded in $H$, we have

$$
\lim _{n}\left\langle a+b\left\|u_{n}\right\|^{2}\right\rangle\left\langle u, u-u_{n}\right\rangle=0
$$

Since $\varepsilon_{n} \rightarrow 0^{+}$, combine (2.9) and (2.10), we conclude that the second term in (2.8) converges to 0 . Hence, $\left\{u_{n}\right\}$ converges strongly to $u$ in $H$. The proof is completed.

Lemma 2.5. Assume that $\left(\mathrm{f}_{1}\right)$ and $\left(\mathrm{f}_{4}\right)$ hold. Then I satisfies the $(P S Z)_{c}$ for every $c \in \mathbb{R}$.

Proof. Let $\left\{u_{n}\right\} \subset H$ satisfies (2.5) and (2.6). It is clear that $\left\{u_{n}\right\}$ is in $K$. Next, we prove that $\left\{u_{n}\right\}$ is bounded in $H$. Suppose to the contrary that $\left\|u_{n}\right\| \rightarrow \infty$. Setting $v_{n}=\frac{u_{n}}{\left\|u_{n}\right\|}$, then $\left\|v_{n}\right\|=1$. So, up to a subsequence, we may assume that

$$
\begin{aligned}
& v_{n} \rightarrow v \quad \text { in } H ; \\
& v_{n} \rightarrow v \quad \text { in } L^{p}(\Omega), \quad \text { for } 1 \leq p<2^{*} ; \\
& v_{n}(x) \rightarrow v(x) \quad \text { a.e. } x \in \Omega .
\end{aligned}
$$

There are two case need to be considered: $v \neq 0$ or $v=0$. We first consider the case $v \neq 0$. Set

$$
\Lambda_{n}\left(r_{1}, r_{2}\right)=\left\{x \in \Omega: r_{1} \leq\left|u_{n}(x)\right|<r_{2}\right\},
$$

and

$$
A:=\{x \in \Omega: v(x) \neq 0\} .
$$

Obviously, meas $(A)>0$. For $x \in A$, we have $\left|u_{n}(x)\right| \rightarrow+\infty$ as $n \rightarrow \infty$. Hence, $A \subset \Lambda_{n}\left(r_{0}, \infty\right)$ for large $n \in \mathbb{N}$, where $r_{0}$ is given in $\left(\mathrm{f}_{2}\right)$. By $\left(\mathrm{f}_{2}\right)$, we have

$$
\frac{F\left(x, u_{n}\right)}{\left|u_{n}\right|^{4}}\left|v_{n}\right|^{4} d x \rightarrow+\infty \quad \text { as } \quad n \rightarrow \infty .
$$


Hence, using Fatou's lemma, we have

$$
\int_{A} \frac{F\left(x, u_{n}\right)}{\left|u_{n}\right|^{4}}\left|v_{n}\right|^{4} d x \rightarrow+\infty \quad \text { as } \quad n \rightarrow \infty .
$$

It follows from (2.5) and (2.11) that

$$
\begin{aligned}
0= & \lim _{n \rightarrow+\infty} \frac{c+o(1)}{\left\|u_{n}\right\|^{4}}=\lim _{n \rightarrow+\infty} \frac{\phi\left(u_{n}\right)}{\left\|u_{n}\right\|^{4}} \\
= & \lim _{n \rightarrow+\infty} \frac{1}{\left\|u_{n}\right\|^{4}}\left(\frac{a}{2}\left\|u_{n}\right\|^{2}+\frac{b}{4}\left\|u_{n}\right\|^{4}-\int_{\Omega} F\left(x, u_{n}\right) d x\right) \\
= & \frac{b}{4}-\lim _{n \rightarrow+\infty} \frac{1}{\left\|u_{n}\right\|^{4}}\left(\int_{\Lambda_{n}\left(0, r_{0}\right)} F\left(x, u_{n}\right) d x+\int_{\Lambda_{n}\left(r_{0}, \infty\right)} F\left(x, u_{n}\right) d x\right) \\
\leq & \frac{b}{4}+\limsup _{n \rightarrow \infty}\left[\frac{c}{\left\|u_{n}\right\|^{3}}\left(1+\frac{r_{0}^{p-1}}{p}\right) \int_{\Omega}\left|v_{n}\right| d x\right] \\
& -\lim _{n \rightarrow+\infty} \int_{A} \frac{F\left(x, u_{n}\right)}{\left|u_{n}\right|^{4}\left|v_{n}\right|^{4} d x} \\
\leq & \frac{b}{4}-\liminf _{n \rightarrow+\infty} \int_{A} \frac{F\left(x, u_{n}\right)}{\left|u_{n}\right|^{4}}\left|v_{n}\right|^{4} d x=-\infty,
\end{aligned}
$$

which is a contradiction, thus $\left\{u_{n}\right\}$ is bounded. By a similar argument as the proof of Lemma 2.4, we can conclude that $\left\{u_{n}\right\}$ converge strongly in $H$.

Next we consider the case $v=0$. We define

$$
\phi\left(t_{n} u_{n}\right)=\max _{t \in[0,1]} \phi\left(t u_{n}\right) .
$$

For any $M>0$, set $\widetilde{v}_{n}=\sqrt{\frac{4 M}{a}} \frac{u_{n}}{\left\|u_{n}\right\|}=\sqrt{\frac{4 M}{a}} v_{n}$. By $\left(\mathrm{f}_{1}\right)$ and he Sobolev embedding theorem, we have

$$
\left|\int_{\Omega} F\left(x, \widetilde{v}_{n}\right) d x\right| \leq c \int_{\Omega}\left|\widetilde{v}_{n}\right| d x+\frac{c}{p} \int_{\Omega}\left|\widetilde{v}_{n}\right|^{p} d x \rightarrow 0,
$$


as $n \rightarrow \infty$. Consequently, for $n$ sufficiently large such that

$$
\phi\left(t_{n} u_{n}\right) \geq \phi\left(\widetilde{v}_{n}\right) \geq \frac{a}{2}\left\|\widetilde{v}_{n}\right\|^{2}+\frac{b}{4}\left\|\widetilde{v}_{n}\right\|^{4}-\int_{\Omega} F\left(x, \widetilde{v}_{n}\right) \geq M .
$$

This means that

$$
\lim _{n \rightarrow \infty} \phi\left(t_{n} u_{n}\right)=\infty
$$

In view of the choice of $t_{n}$ we know that $\left\langle\phi^{\prime}\left(t_{n} u_{n}\right), t_{n} u_{n}\right\rangle=0$. Hence, by $\left(\mathrm{f}_{4}\right)$, we have

$$
\begin{aligned}
\infty & \leftarrow \phi\left(t_{n} u_{n}\right)-\frac{1}{4}\left\langle\phi^{\prime}\left(t_{n} u_{n}\right), t_{n} u_{n}\right\rangle=\frac{a}{4}\left\|t_{n} u_{n}\right\|^{2}+\int_{\Omega} \widetilde{F}\left(x, t_{n} u_{n}\right) d x \\
& \leq \frac{a}{4}\left\|u_{n}\right\|^{2}+\int_{\Omega} \widetilde{F}\left(x, u_{n}\right) d x=\phi\left(u_{n}\right)-\frac{1}{4}\left\langle\phi^{\prime}\left(u_{n}\right), u_{n}\right\rangle,
\end{aligned}
$$

which contradicts (2.5), thus $\left\{u_{n}\right\}$ is bounded in $H$. Since $\left\{u_{n}\right\} \subset H$ is bounded, using a similar arguments as (2.8), (2.9), and (2.10), we can conclude that $u_{n} \rightarrow u$ in $H$, as $n \rightarrow \infty$. This completes the proof.

\section{Proof of Main Results}

Let $\left\{e_{j}\right\}$ is an orthonormal basis of $H$ and define $x_{j}=\mathbb{R} e_{j}$,

$$
Y_{l}=\bigoplus_{j=1}^{l} X_{j}, \quad Z_{k}=\bigoplus_{j=k}^{\infty} X_{j}, \quad l, k \in \mathbb{Z}
$$

Therefore, we have the following lemma from [9].

Lemma 3.1 ([9], Lemma 3.8). If $1 \leq p<2^{*}$, then we have

$$
\beta_{k}(p):=\sup _{u \in Z_{k},\|u\|=1}|u|_{p} \rightarrow 0, \quad k \rightarrow \infty .
$$

Lemma 3.2. Suppose that $\left(\mathrm{f}_{1}\right)$ is satisfied. Then there exist constants $\rho, \alpha>0$ and $m \in \mathbb{Z}$ such that $\left.I\right|_{\partial B_{\rho} \cap Z_{m}} \geq \alpha$. 
Proof. By Lemma 3.1, we can choose an integer $m \geq 1$ such that $0<\beta_{m}(1)<<1,0<\beta_{m}(p)<<1$ and

$$
\|u\|_{1} \leq \beta_{m}(1)\|u\|, \quad\|u\|_{p} \leq \beta_{m}(p)\|u\|, \quad \forall u \in X_{1} .
$$

For any $u \in Z_{m}$ with $\|u\|=\rho<1$, by $\left(\mathrm{f}_{1}\right)$, we have

$$
\begin{aligned}
I(u) & =\phi(u)=\frac{a}{2}\|u\|^{2}+\frac{b}{4}\|u\|^{4}-\int_{\Omega} F(x, u) d x \\
& \geq \frac{a}{2}\|u\|^{2}-c \int_{\Omega}|u| d x-\frac{c}{p} \int_{\Omega}|u|^{p} d x \\
& \geq \frac{a}{2}\|u\|^{2}-c \beta_{m}(1)\|u\|-\frac{c}{p} \beta_{m}^{p}(p)\|u\|^{p} \\
& \geq \frac{a}{2}\|u\|^{2}-c \beta_{m}(1)\|u\|-c_{p}\|u\|^{p} \\
& =\rho\left(\frac{a}{2} \rho-c \beta_{m}(1)-c_{p} \rho^{p-1}\right)>0 .
\end{aligned}
$$

Here we use the fact that $0<\beta_{m}(1)<\frac{a}{2 c} \rho-\frac{c_{p}}{c} \rho^{p-1}$ if $m$ suitable large. Thus, this completes the proof.

Lemma 3.3. Suppose that $\left(\mathrm{f}_{1}\right)$ and $\left(\mathrm{f}_{2}\right)$ are satisfied. Then, for any finite dimensional subspace $\tilde{H} \subset H$, there is $R=R(\widetilde{H})>0$ such that

$$
\phi(u) \leq 0, \quad \forall u \in \tilde{H} \backslash B_{R} .
$$

Proof. For any finite dimensional subspace $\tilde{H} \subset H$, there is a positive integral number $n>m$ (where $m$ is given by Lemma 3.2) such that $\widetilde{H} \subset Y_{n}$. Since all norms are equivalent in a finite dimensional space, there is a constant $c_{4}>0$ such that

$$
\|u\|_{4} \geq c_{4}\|u\|, \quad \forall Y_{n} .
$$


By $\left(\mathrm{f}_{1}\right)$ and $\left(\mathrm{f}_{2}\right)$, we know that for any $M>\frac{b}{4 c_{4}^{4}}$ there is a constant $C_{M}>0$ such that

$$
F(x, u) \geq M|u|^{4}-C_{M}|u|^{2}, \quad \forall(x, u) \in \Omega \times \mathbb{R} .
$$

It follows from (3.2) and (3.3) that

$$
\begin{aligned}
I(u) & =\phi(u) \leq \frac{1}{2}\|u\|^{2}+\frac{b}{4}\|u\|^{4}-M\|u\|_{4}^{4}+C_{M}\|u\|_{2}^{2} \\
& \leq \frac{1}{2}\|u\|^{2}-\left(M c_{4}^{4}-\frac{b}{4}\right)\|u\|^{4}+C_{M} C_{2}^{2}\|u\|^{2},
\end{aligned}
$$

for all $u \in Y_{n}$. Consequently, there is a large $R=R(\tilde{H})>0$ such that $\phi(u) \leq 0$ on $\tilde{H} \backslash B_{R}$. Thus, the proof is complete.

Proof of Theorem 1.1. Let $X=H, X_{1}=Z_{m}$, and $X_{2}=Y_{n}$. Obviously, $I(0)=0$ and $\left(f_{5}\right)$ implies that $I$ is even. By Lemmas 2.4, 3.1, and 3.2, all conditions of Theorem 2.2 are satisfied. Thus, problem (2.5) possesses $\operatorname{dim} X_{2}-\operatorname{codim} X_{1}=n-m+1>1$ distinct pairs of positive solutions.

Proof of Theorem 1.2. Let $X=H, X_{1}=Z_{m}$, and $X_{2}=Y_{n}$. Obviously, $I(0)=0$ and $\left(f_{5}\right)$ implies that $I$ is even. By Lemmas 2.5, 3.1, and 3.2, all conditions of Theorem 2.2 are satisfied. Thus, problem (2.5) possesses $\operatorname{dim} X_{2}-\operatorname{codim} X_{1}=n-m+1>1$ distinct pairs of positive solutions.

\section{References}

[1] A. Friedman, Variational Principles and Free Boundary Value Problems, WileyInterscience, New York, 1983.

[2] J. Heinonen, T. Kilpelainen and O. Martio, Nonlinear Potential Theory of Degenerate Elliptic Equations, Oxford Univ. Press, Oxford, 1993. 
[3] S. Karamardian, Generalized complementarity problem, J. Opim. Theory Appl. 8(3) (1971), 161-168.

DOI: https://doi.org/10.1007/BF00932464

[4] D. Kinderlehrer and G. Stampacchia, Convex Programming and Variational Inequalities and their Applications, Academic Press, New York, 1980.

[5] O. Mancino and G. Stampacchia, Convex programming and variational inequalities, J. Optim. Theory Appl. 9(1) (1972), 3-23.

DOI: https://doi.org/10.1007/BF00932801

[6] J. F. Rodrigues, Obstacle problems in mathematical physics, In: Mathematics Studies, 134, Elsevier (1987).

[7] A. Szulkin, Minimax principles for lower semicountinuous functions and application to nonlinear boundary value problems, Ann. Inst. H. Poincaré Anal. Nonlinéaire 3(2) (1986), 77-109.

[8] G. M. Troianiello, Elliptic Differential Equations and Obstacle Problems, The University Series in Mathematics, 1987.

[9] M. Willem, Minimax Theorems, Birkhäuser, Berlin, 1996.

[10] A. Ambrosetti and P. H. Rabinowitz, Dual variational methods in critical point theory and applications, J. Funct. Anal. 14(4) (1973), 349-381.

DOI: https://doi.org/10.1016/0022-1236(73)90051-7 